\title{
Swarm Intelligence in Data Science: Applications, Opportunities and Challenges
}

\author{
Jian Yang ${ }^{1}$, Liang $\mathrm{Qu}^{1}$, Yang Shen ${ }^{1}$, Yuhui Shi ${ }^{1(凶)}$, Shi Cheng ${ }^{2}$, \\ Junfeng Zhao ${ }^{3}$, and Xiaolong Shen ${ }^{3}$ \\ 1 Department of Computer Science and Engineering, \\ Southern University of Science and Technology, Shenzhen 518055, China \\ shiyh@sustech.edu.cn \\ 2 School of Computer Science, Shaanxi Normal University, Xi'an 710119, China \\ 32012 Laboratories, Huawei Technologies Co., Ltd., Shenzhen 518129, China
}

\begin{abstract}
The Swarm Intelligence (SI) algorithms have been proved to be a comprehensive method to solve complex optimization problems by simulating the emergence behaviors of biological swarms. Nowadays, data science is getting more and more attention, which needs quick management and analysis of massive data. Most traditional methods can only be applied to continuous and differentiable functions. As a set of population-based approaches, it is proven by some recent research works that the SI algorithms have great potential for relevant tasks in this field. In order to gather better insight into the utilization of these methods in data science and to provide a further reference for future researches, this paper focuses on the relationship between data science and swarm intelligence. After introducing the mainstream swarm intelligence algorithms and their common characteristics, both the theoretical and real-world applications in the literature which utilize the swarm intelligence to the related domains of data analytics are reviewed. Based on the summary of the existing works, this paper also analyzes the opportunities and challenges in this field, which attempts to shed some light on designing more effective algorithms to solve the problems in data science for real-world applications.
\end{abstract}

Keywords: Swarm Intelligence $\cdot$ Data science $\cdot$ Evolutionary computation · Unified Swarm Intelligence

This work is partially supported by National Key R\&D Program of China under the Grant No. 2017YFC0804003, National Science Foundation of China under grant number 61761136008, 61806119, Shenzhen Peacock Plan under Grant No. KQTD2016 112514355531, Program for Guangdong Introducing Innovative and Entrepreneurial Teams under grant number 2017ZT07X386, the Science and Technology Innovation Committee Foundation of Shenzhen under the Grant No. ZDSYS201703031748284, Natural Science Basic Research Plan In Shaanxi Province of China under grant No. 2019JM-320, Huawei HIRP Open Project under grant No. HO2019040806003P118, Guangdong Provincial Key Laboratory under Grant No. 2020B121201001, and SUSTech Artificial Intelligence Institute (SAINT).

(C) Springer Nature Switzerland AG 2020

Y. Tan et al. (Eds.): ICSI 2020, LNCS 12145, pp. 3-14, 2020.

https://doi.org/10.1007/978-3-030-53956-6_1 


\section{Introduction}

Data science has been widely concerned in recent years. One of the most important aspects of data science is data analytics, which aims to automatic extraction of knowledge from massive data. Traditional model-based methods are mainly on fitting the collected data to some predefined mathematical models. However, these models may fail when encountering problem varieties such as the volume, the dynamical changes, noise, and so forth. With the increase of the above varieties, traditional data processing approaches will become inefficient or even ineffective. Because of the above difficulties, new and efficient methods should be developed to deal with data analysis tasks [11]. Now the mainstream methods are shifting from traditional model-driven to data-driven paradigms. Many applications in data science can be transferred to optimization problems. Thus it requires the algorithms to have the ability to search the solution space and find the optimums [9]. Traditional model-based methods need the problems that can be written into the form of continuous and differentiable functions. However, in the face of a large amount of data and complex tasks, it is often difficult to achieve.

The population-based meta-heuristic algorithms are good at solving those problems, which the traditional methods can not deal with or, at least, be challenging to solve [10]. Swarm Intelligence (SI), a kind of meta-heuristic algorithms, is attracting more and more attention and has been proven to be sufficient to handle the large scale, dynamic, multi-objective problems in data analytics. As shown in Fig. 1, there are mainly two categories of approaches that utilize SI algorithms in data science [41]. The first approach uses swarm intelligence as a parameter tuning/optimizing method of data mining technologies may including machine learning, statistics, and others. The second category directly applies the SI algorithms on data organization, i.e., move data instances place on a lowdimensional feature space to reach a suitable clustering or reduce the dimensionality of the data.

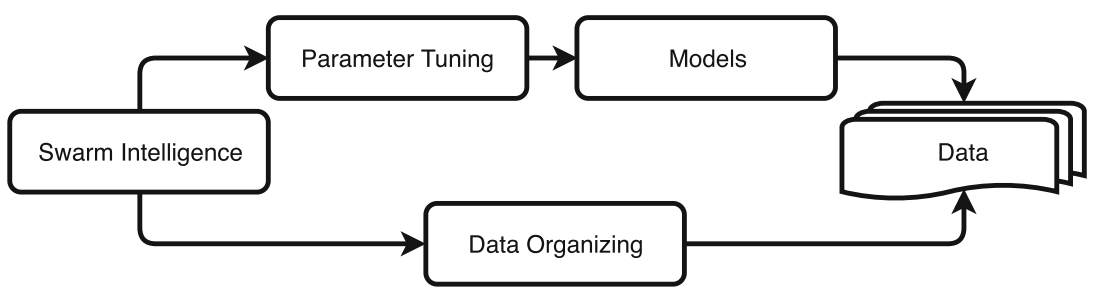

Fig. 1. Two approaches of Swarm Intelligence for data science

Swarm Intelligence is a group of nature-inspired searching and optimization techniques that studies collective intelligence in a population of low complexity individuals [32]. The SI algorithms are inspired by the interactions among 
individuals within a group or several groups, which involves the patterns of competition and cooperation [16]. SI algorithms use a population of individuals to search in a problem domain. Each individual represents a potential solution for the problem being optimized. During a guided search process, SI algorithms maintain and improve a collection of potential solutions successively until some predefined stopping condition is met, i.e., either the result is acceptable, or the number of iterations is reached [26].

In order to gather better insight into the utilization of these methods in data science and to provide a further reference for future researches, this paper focuses on the data science related works that utilizing swarm intelligence in the past few years. After introducing the mainstream swarm intelligence algorithms and their common characteristics, both the theoretical and real-world applications in the literature which utilize the swarm intelligence to the related domains of data analytics are reviewed. Based on the summary of the existing works, this paper also analyzes the opportunities and challenges in this field, which attempts to shed some light on designing more effective algorithms to solve the problems in data science for real-world applications. The remaining of the paper is organized as follows. Section 2 briefly reviews the development of swarm intelligence and some major algorithms in this field. Section 3 introduces some theoretical applications in the literature that adopt swarm intelligence algorithms in data science. Section 4 gives a set of real-world applications. The opportunities and challenges of applying SI algorithms to data science are discussed in Sect. 5, followed by the conclusions reached in Sect. 6 .

\section{Swarm Intelligence Algorithms}

\subsection{General Procedure of SI Algorithms}

SI Algorithms is a set of artificial intelligence techniques inspired by biological swarm behaviors at both macro and micro levels. They generally have selforganizing and decentralizing paradigms with the characteristics of scalability, adaptability, robustness, and individual simplicity. In SI algorithms, a population of individuals, which indicates potential candidate solutions, cooperating among themselves and statistically becoming better and better over iterations, then eventually finding good enough solutions [45]. In recent years, a large number of swarm intelligence methods have been proposed. These methods have different inspiration sources and various operations. In general, these different operations are trying to balance the convergence and diversity of the search process, i.e., the balance between exploration and exploitation.

The general procedure of swarm intelligence algorithms can be summarized in Algorithm 1. Starting from the random initialization of a population of individuals in solution space, followed by the corresponding evaluation process and new solution generation process, after a certain number of iterations, swarm intelligence algorithms can eventually find acceptable solutions.

As a general principle, the expected fitness value of a solution should improve as more computational resources in time and/or space are given. More desirable, 


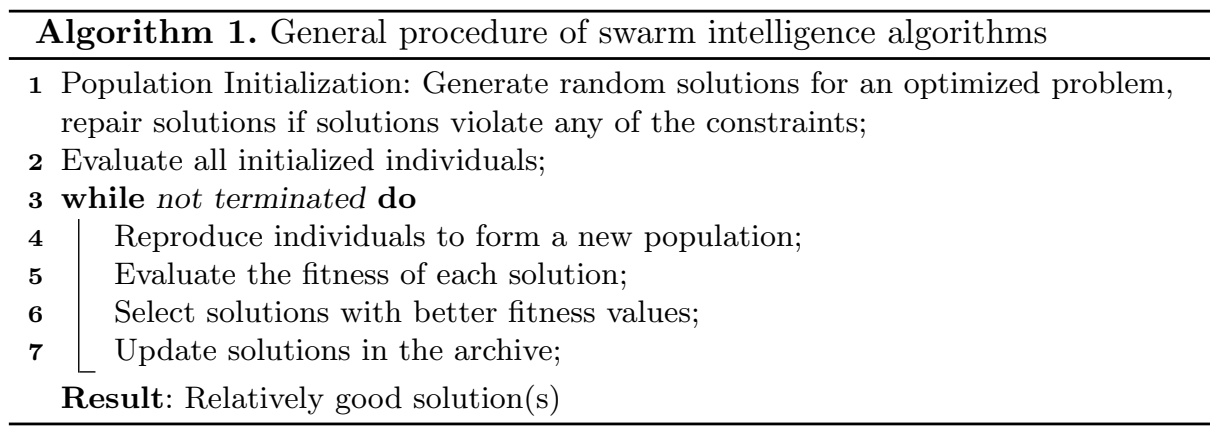

the quality of the solution should improve monotonically over iterations, i.e., the fitness value of the solution at time $t+1$ should be no worse than the fitness at time $t$.

\subsection{Developments}

In the past 30 years, a large number of swarm intelligence algorithms have emerged. They get inspiration from different phenomena, and design corresponding new solution generation operations with the considerations of balancing convergence and diversity of the swarm. As shown in Table 1, the source of inspirations are varying from human society (BSO, TLBO), animals (BA, GWO, MA, LOA), insects and birds (PSO, ACO, ABC, FA, CS, GSO), bacterias (BFO), and also some human-made phenomenon (FWA).

With the increasing prominence of NP-hard problems, it is almost impossible to find the optimal solutions in real-time. The number of potential solutions to these problems is often infinite. In this case, it is essential to find a feasible solution within the time limit. SI algorithms have found its practicability in the practical application of solving nonlinear problems in almost all fields of science, engineering, and industrial fields: From data mining to optimization, computational intelligence, business planning, bioinformatics, as well as industrial applications. Now is the era of big data, those mentioned above scientific and engineering problems, more or less, are related to data issues. Swarm intelligence has made a lot of successful applications in data relevant applications. Meanwhile, with the increasing dynamics, noises, and complexity of tasks, there still are many opportunities along with challenges in the applications of swarm intelligence with data sciences.

\section{Theoretical Applications}

For decades, data mining has been a hot academic topic in the field of computer science statistics. As mentioned, the SI algorithm is mainly used in data mining tasks in two forms: parameter tuning or data organizing. Main applications, including dimensionality reduction, classification, and clustering, as well as automated machine learning. 
Table 1. Some Swarm Intelligence algorithms with source of inspiration

\begin{tabular}{|c|c|c|}
\hline Algorithms & Source of inspiration & \\
\hline $\begin{array}{l}\text { Brain Storm Opt., BSO [49] } \\
\text { Teaching-learning-based Opt., TLBO [48] }\end{array}$ & $\begin{array}{l}\text { Brainstorming process of human } \\
\text { How teachers influence learners }\end{array}$ & Human society \\
\hline $\begin{array}{l}\text { Particle Swarm Opt., PSO [31] } \\
\text { Ant Colony Opt., ACO [15] } \\
\text { Artificial Bee Colony, ABC [29] } \\
\text { Firefly Algorithms, FA [66] } \\
\text { Glowworm Swarm Opt., GSO [36] } \\
\text { Cuckoo Search, CS [67] }\end{array}$ & $\begin{array}{l}\text { Bird flocking and foraging } \\
\text { Ants foraging mechanisms } \\
\text { Foraging behavior of honey bees } \\
\text { Bioluminescence of fireflies } \\
\text { Luciferin induced glow of a glowworm } \\
\text { Obligate brood parasitism in cuckoos }\end{array}$ & Insects, birds, etc. \\
\hline $\begin{array}{l}\text { Bat Algorithm, BA [68] } \\
\text { Grey Wolf Opt., GWO [42] } \\
\text { Monkey Algorithm, MA [73] } \\
\text { Lion Opt. Algorithm, LOA [69] }\end{array}$ & $\begin{array}{l}\text { Echolocation behaviors of micro-bats } \\
\text { Leadership and hunting of grey wolves } \\
\text { Climbing techniques used by monkeys } \\
\text { Cooperation characteristics of lions }\end{array}$ & Animals \\
\hline Bacterial Foraging Opt, BFO [13] & Group foraging behavior of bacteria & Microscopic \\
\hline Fireworks Algorithm, FWA [57] & Fireworks explosion & Other \\
\hline
\end{tabular}

\subsection{Dimensionality Reduction}

Dimensionality reduction is the process of reducing the number of random variables or attributes in a dataset under consideration. It plays a vital role in data preprocessing for data mining. There are generally two operations for dimensionality reduction: feature selection and feature extraction. Feature selection is a process of selecting an optimal subset of relevant features for use in model construction. While feature extraction is a process of project original data in a high dimensional space onto a smaller space. The accuracy of a model will be enhanced by using wisely selected/projected features rather than all available features in a large amount of data.

Since feature selection is an NP-hard combinatorial optimization problem, SI algorithms are found to be a promise option to solve those kinds of problems. A lot of related works has emerged recently, the following are some examples: $\mathrm{Gu}$ et al. proposed a feature selection method for high dimensional classification based on a very recent PSO variant, known as Competitive Swarm Optimizer (CSO) [23]. Hang et al. designed an FA based method for feature selection, which has the ability to prevent premature convergence [72]. Pourpanah et al. combine the Fuzzy ARTMAP (FAM) model with the BSO algorithm for feature selection tasks [47], etc. A more detailed survey about SI powered feature selection can be found in [44].

\subsection{Classification and Clustering}

Classification and clustering are essential aspects of data science. They have been studied widely in the domain of statistics, neural networks, machine learning, and knowledgeable systems over the decades. In general, classification is to predict the target class by analyzing the training dataset, while clustering is to group the similar kind of targets by considering the most satisfying condition. 
The SI applications in those two aspects are mainly related to parameter tuning. For classification, works can be found in literature that combine SI algorithms with regression model [53], support vector machine [7,14,60], k-nearest neighbor classifiers $[58,65]$, Decision trees [3,35], as well as the neural networks $[30,62]$. For clustering, some recent works are related to utilizing SI with k-means [28,59,61], c-means [21], and other linear or non-linear clustering algorithms $[19,27]$.

\subsection{Automated Machine Learning}

In the past decade, the research and application of machine learning have seen explosive growth, especially the Deep neural networks (DNNs) [37] has made great progress in many application fields. However, the performance of many machine learning methods is very sensitive to too many design decisions. In particular, the architecture designing of DNNs is very complex and highly rely on the experts' prior knowledge. To address this problem, many SI based methods are proposed to automatically design DNNs [54].

Wang et al. [64] propose an efficient particle swarm optimisation (EPSOCNN) approach to automatically design the architectures of convolutional neural networks (CNNs). Specifically, in order to reduce the computation cost, EPSOCNN minimises the hyperparameter space of CNNs to a single block and evaluates the candidate CNNs with the small subset of the training set. Wang et al. [63] propose a multi-objective evolutionary CNNs (MOCNN) to search the nondominant CNN architectures at the Pareto front in terms of the classification accuracy objective and the computational cost objective. It introduces a novel encoding strategy to encode CNNs and utilizes a multi-objective particle swarm optimization (OMOPSO) to optimize the candidate CNNs architectures.

\section{Real-World Applications}

Social Community Network Analysis. Social network analysis plays an important role in many real-world problems, such as the community detection techniques $[20,46]$ which aims to mine the implicit community structures in the networks. Recently, many SI methods have shown a promising potential in many community detection problems. Lyu et al. [40] propose a novel local community detection method called evolutionary-based local community detection (ECLD), which utilizes the entire obtained information and PSO algorithm to find the local community structures in the complex networks. Sun et al. [55] introduce a Parallel Self-organizing Overlapping Community Detection (PSOCD) method inspired by the swarm intelligence system to detect the overlapping communities in the large scale dynamic complex networks. It treats the complex networks as a decentralized, self-organized, and self-evolving system. They can iteratively find the community structures. Other releavant works can be refer to $[6,22,25]$. 
Scheduling and Routing. Scheduling and routing problems are very common in real world, as long as there are resources to manage. For example, the PSO algorithm was used in power systems for demand response management [17], consumer demand management [38], etc.

Internet of Things. Internet of Things (IoT) is another real-world application in which SI algorithms have been widely used [5]. For example, in IoT-based systems, the SI algorithm has been used for task scheduling [4]. In IoT-based smart cities, SI algorithms have been used due to its population-based feature to make the system flexible and scalable [70].

Bioinformatics is an interdisciplinary field that develops algorithms and software tools for processing biological data samples. Various biological problems could be represented as an optimization problem and solved by SI algorithms. For example, the protein design problem could be represented as a combinatorial optimization problem [24]. More information is summarized in [56].

Resource Allocation. Resource allocation is the process of allocating and managing assets in an optimized way to support the strategic objectives of an organization. SI algorithms have been used in many related applications such as Cloud service resource allocation [8], wireless network planning [2], etc.

Others. Apart from the real-world applications discussed above, SI algorithms have also been applied to many other real-world systems that are data related. For example, the wind farm decision system [74] to reduce the cost of wind farms, autonomous DDoS attack detection [33], anomaly intrusion detection [18], image analysis [34,51], facial recognition [43], Medical Image Segmentation [52], and natural language processing $[1,39]$, etc.

\section{Opportunities and Challenges}

Unified Swarm Intelligence. Unified Swarm Intelligence Are there any universal rules behind this growing field? What are the fundamental components of a good swarm intelligence algorithm to have? There are dozens of SI algorithms proposed so far and sharing similar operations on solving problems. Is there a unified framework for SI algorithms that has the ability to develop its learning capacity that can better solve an optimization problem which is unknown at the algorithms design or implementation time [50]. How to correctly identify and extract the fundamental components of SI algorithms, so that they can form new algorithms automatically according to the character of the problem on hand, is a challenge. Some efforts are trying to solve this problem [12,50,71], but more work is needed to make it a reality. 
Handling High Dimensional and Dynamical Data. The "curse of dimensionality" happens on high-dimensional data mining problems when the dimension of the data space increases. For example, the nearest neighbor approaches are instrumental in categorization. However, for high dimensional data, it is complicated to solve the similarity search problem due to the computational complexity, which was caused by the increase of dimensionality. Furthermore, when the problems are in non-stationary environments, or uncertain environments, i.e., the conditions of data dynamically change over time, additional measures must be taken, so that swarm intelligence algorithms are still able to solve satisfactorily dynamic problems.

SI Based AutoML. As mentioned before, swarm intelligence algorithms can not only be used for automatic optimization of hyper-parameters of the machine learning model, but also the automated design of the model structure. With the development of AutoML, the swarm intelligence algorithm has great potential in this field. However, in addition to hyper-parameter optimization, the representation of learning model and the mechanism of model evaluation are also come with challenges.

\section{Conclusion}

This paper has reviewed related works that applying swarm intelligence algorithms in data science. The fundamentals and developments of swarm intelligence are briefly summarized. The theoretical applications such as SI based dimensionality reduction, classification, clustering, as well as automated machine learning are also introduced. A short review of real-world applications, including social community network analysis, scheduling and routing, internet of things, bioinformatics, and resource allocation, are also given, then followed by the opportunities and challenges in this field. Generally speaking, the swarm intelligence algorithm has been widely used in the field of data science in the past decades, including theoretical and practical applications. Moreover, with the development of artificial intelligence technology and data science, swarm intelligence algorithms have great opportunities in different aspects of data science. Nevertheless, it also faces a series of challenges, which need more in-depth research.

\section{References}

1. Abualigah, L.M., Khader, A.T., Hanandeh, E.S.: A new feature selection method to improve the document clustering using particle swarm optimization algorithm. J. Comput. Sci. 25, 456-466 (2018)

2. Ari, A.A.A., Gueroui, A., Titouna, C., Thiare, O., Aliouat, Z.: Resource allocation scheme for 5G C-RAN: a swarm intelligence based approach. Comput. Netw. 165, 106957 (2019)

3. Bida, I., Aouat, S.: A new approach based on bat algorithm for inducing optimal decision trees classifiers. In: Rocha, Á., Serrhini, M. (eds.) EMENA-ISTL 2018. SIST, vol. 111, pp. 631-640. Springer, Cham (2019). https://doi.org/10.1007/9783-030-03577-8_69 
4. Boveiri, H.R., Khayami, R., Elhoseny, M., Gunasekaran, M.: An efficient swarmintelligence approach for task scheduling in cloud-based internet of things applications. J. Ambient Intell. Humaniz. Comput. 10(9), 3469-3479 (2019)

5. Chakraborty, T., Datta, S.K.: Application of swarm intelligence in internet of things. In: 2017 IEEE International Symposium on Consumer Electronics (ISCE), pp. 67-68. IEEE (2017)

6. Honghao, C., Zuren, F., Zhigang, R.: Community detection using ant colony optimization. In: 2013 IEEE Congress on Evolutionary Computation, Cancun, Mexico, pp. 3072-3078. IEEE (2013)

7. Chen, H.L., Yang, B., Wang, G., Wang, S.J., Liu, J., Liu, D.Y.: Support vector machine based diagnostic system for breast cancer using swarm intelligence. J. Med. Syst. 36(4), 2505-2519 (2012)

8. Cheng, S., et al.: Cloud service resource allocation with particle swarm optimization algorithm. In: He, C., Mo, H., Pan, L., Zhao, Y. (eds.) BIC-TA 2017. CCIS, vol. 791, pp. 523-532. Springer, Singapore (2017). https://doi.org/10.1007/978-981-107179-9_41

9. Cheng, S., Liu, B., Shi, Y., Jin, Y., Li, B.: Evolutionary computation and big data: key challenges and future directions. In: Tan, Y., Shi, Y. (eds.) DMBD 2016. LNCS, vol. 9714, pp. 3-14. Springer, Cham (2016). https://doi.org/10.1007/9783-319-40973-3_1

10. Cheng, S., Liu, B., Ting, T., Qin, Q., Shi, Y., Huang, K.: Survey on data science with population-based algorithms. Big Data Anal. 1(1), 3 (2016)

11. Cheng, S., Shi, Y., Qin, Q., Bai, R.: Swarm intelligence in big data analytics. In: Yin, H., et al. (eds.) IDEAL 2013. LNCS, vol. 8206, pp. 417-426. Springer, Heidelberg (2013). https://doi.org/10.1007/978-3-642-41278-3_51

12. Chu, X., Wu, T., Weir, J.D., Shi, Y., Niu, B., Li, L.: Learning-interactiondiversification framework for swarm intelligence optimizers: a unified perspective. Neural Comput. Appl. 32, 1-21 (2018). https://doi.org/10.1007/s00521-018-3657-0

13. Das, S., Biswas, A., Dasgupta, S., Abraham, A.: Bacterial foraging optimization algorithm: theoretical foundations, analysis, and applications. In: Abraham, A., Hassanien, A.E., Siarry, P., Engelbrecht, A. (eds.) Foundations of Computational Intelligence, vol. 3, pp. 23-55. Springer, Heidelberg (2009). https://doi.org/10. 1007/978-3-642-01085-9_2

14. Ding, S., An, Y., Zhang, X., Wu, F., Xue, Y.: Wavelet twin support vector machines based on glowworm swarm optimization. Neurocomputing 225, 157-163 (2017)

15. Dorigo, M., Birattari, M., Stutzle, T.: Ant colony optimization. IEEE Comput. Intell. Mag. 1(4), 28-39 (2006)

16. Eberhart, R.C., Shi, Y., Kennedy, J.: Swarm Intelligence. Elsevier, London (2001)

17. Faria, P., Vale, Z., Soares, J., Ferreira, J.: Demand response management in power systems using particle swarm optimization. IEEE Intell. Syst. 28(4), 43-51 (2011)

18. Feng, Y., Wu, Z.F., Wu, K.G., Xiong, Z.Y., Zhou, Y.: An unsupervised anomaly intrusion detection algorithm based on swarm intelligence. In: 2005 International Conference on Machine Learning and Cybernetics, vol. 7, pp. 3965-3969. IEEE (2005)

19. Figueiredo, E., Macedo, M., Siqueira, H.V., Santana Jr., C.J., Gokhale, A., BastosFilho, C.J.: Swarm intelligence for clustering-a systematic review with new perspectives on data mining. Eng. Appl. Artif. Intell. 82, 313-329 (2019)

20. Fortunato, S.: Community detection in graphs. Phys. Rep. 486(3-5), 75-174 (2010). arXiv:0906.0612 
21. Fuchs, C., Spolaor, S., Nobile, M.S., Kaymak, U.: A swarm intelligence approach to avoid local optima in fuzzy c-means clustering. In: 2019 IEEE International Conference on Fuzzy Systems (FUZZ-IEEE), pp. 1-6. IEEE (2019)

22. Ghasabeh, A., Abadeh, M.S.: Community detection in social networks using a hybrid swarm intelligence approach. Int. J. Knowl. Based Intell. Eng. Syst. 19(4), 255-267 (2015). IOS Press

23. Gu, S., Cheng, R., Jin, Y.: Feature selection for high-dimensional classification using a competitive swarm optimizer. Soft. Comput. 22(3), 811-822 (2018)

24. Hallen, M.A., Donald, B.R.: Protein design by provable algorithms. Commun. ACM 62(10), 76-84 (2019)

25. Hassan, E.A., Hafez, A.I., Hassanien, A.E., Fahmy, A.A.: Community detection algorithm based on artificial fish swarm optimization. In: Filev, D., et al. (eds.) Intelligent Systems'2014. AISC, vol. 323, pp. 509-521. Springer, Cham (2015). https://doi.org/10.1007/978-3-319-11310-4_44

26. Hussain, K., Salleh, M.N.M., Cheng, S., Shi, Y.: Metaheuristic research: a comprehensive survey. Artif. Intell. Rev. 52(4), 2191-2233 (2019)

27. Inkaya, T., Kayalıgil, S., Özdemirel, N.E.: Swarm intelligence-based clustering algorithms: a survey. In: Celebi, M., Aydin, K. (eds.) Unsupervised Learning Algorithms, pp. 303-341. Springer, Cham (2016). https://doi.org/10.1007/978-3-31924211-8_12

28. Kang, Q., Liu, S., Zhou, M., Li, S.: A weight-incorporated similarity-based clustering ensemble method based on swarm intelligence. Knowl. Based Syst. 104, 156-164 (2016)

29. Karaboga, D., Basturk, B.: A powerful and efficient algorithm for numerical function optimization: artificial bee colony (ABC) algorithm. J. Global Optim. 39(3), 459-471 (2007). https://doi.org/10.1007/s10898-007-9149-x

30. Karpat, Y., Ozel, T.: Hard Turning Optimization Using Neural Network Modeling and Swarm Intelligence. Society of Manufacturing Engineers, Dearborn (2000)

31. Kennedy, J., Eberhart, R.: Particle swarm optimization. In: Proceedings of ICNN 1995-International Conference on Neural Networks, vol. 4, pp. 1942-1948. IEEE (1995)

32. Kennedy, J., Eberhart, R., Shi, Y.: Swarm Intelligence. Morgan Kaufmann Publisher, San Francisco (2001)

33. Kesavamoorthy, R., Soundar, K.R.: Swarm intelligence based autonomous DDOS attack detection and defense using multi agent system. Cluster Comput. 22(4), 9469-9476 (2019). https://doi.org/10.1007/s10586-018-2365-y

34. Khadhraoui, T., Ktata, S., Benzarti, F., Amiri, H.: Features selection based on modified PSO algorithm for 2D face recognition. In: 2016 13th International Conference on Computer Graphics, Imaging and Visualization (CGiV), pp. 99-104. IEEE (2016)

35. Kozak, J., Boryczka, U.: Collective data mining in the ant colony decision tree approach. Inf. Sci. 372, 126-147 (2016)

36. Krishnanand, K., Ghose, D.: Glowworm swarm optimization for simultaneous capture of multiple local optima of multimodal functions. Swarm Intell. 3(2), 87-124 (2009)

37. LeCun, Y., Bengio, Y., Hinton, G.: Deep learning. Nature 521(7553), 436-444 (2015)

38. Lin, Y.H., Hu, Y.C.: Residential consumer-centric demand-side management based on energy disaggregation-piloting constrained swarm intelligence: towards edge computing. Sensors 18(5), 1365 (2018) 
39. Lu, Y., Liang, M., Ye, Z., Cao, L.: Improved particle swarm optimization algorithm and its application in text feature selection. Appl. Soft Comput. 35, 629-636 (2015)

40. Lyu, C., Shi, Y., Sun, L.: A novel local community detection method using evolutionary computation. IEEE Trans. Cybern., 1-13 (2019). https://doi.org/10.1109/ TCYB.2019.2933041

41. Martens, D., Baesens, B., Fawcett, T.: Editorial survey: swarm intelligence for data mining. Mach. Learn. 82(1), 1-42 (2011). https://doi.org/10.1007/s10994010-5216-5

42. Mirjalili, S., Mirjalili, S.M., Lewis, A.: Grey wolf optimizer. Adv. Eng. Softw. 69, 46-61 (2014)

43. Nebti, S., Boukerram, A.: Swarm intelligence inspired classifiers for facial recognition. Swarm Evol. Comput. 32, 150-166 (2017)

44. Nguyen, B.H., Xue, B., Zhang, M.: A survey on swarm intelligence approaches to feature selection in data mining. Swarm Evol. Comput. 54, 100663 (2020)

45. Panigrahi, B.K., Shi, Y., Lim, M.H.: Handbook of Swarm Intelligence: Concepts, Principles and Applications, vol. 8. Springer, Heidelberg (2011). https://doi.org/ 10.1007/978-3-642-17390-5

46. Pizzuti, C.: Evolutionary computation for community detection in networks: a review. IEEE Trans. Evol. Comput. 22(3), 464-483 (2018)

47. Pourpanah, F., Shi, Y., Lim, C.P., Hao, Q., Tan, C.J.: Feature selection based on brain storm optimization for data classification. Appl. Soft Comput. 80, 761-775 (2019)

48. Rao, R.V., Savsani, V.J., Vakharia, D.: Teaching-learning-based optimization: a novel method for constrained mechanical design optimization problems. Comput. Aided Des. 43(3), 303-315 (2011)

49. Shi, Y.: Brain storm optimization algorithm. In: Tan, Y., Shi, Y., Chai, Y., Wang, G. (eds.) ICSI 2011. LNCS, vol. 6728, pp. 303-309. Springer, Heidelberg (2011). https://doi.org/10.1007/978-3-642-21515-5_36

50. Shi, Y.: Unified swarm intelligence algorithms. In: Shi, Y. (ed.) Critical Developments and Applications of Swarm Intelligence, pp. 1-26. IGI Global, Hershey (2018)

51. Silva, P.H., Luz, E., Zanlorensi, L.A., Menotti, D., Moreira, G.: Multimodal feature level fusion based on particle swarm optimization with deep transfer learning. In: 2018 IEEE Congress on Evolutionary Computation (CEC), pp. 1-8. IEEE (2018)

52. Singh, T.I., Laishram, R., Roy, S.: Comparative study of combination of swarm intelligence and fuzzy $\mathrm{C}$ means clustering for medical image segmentation. In: Luhach, A., Hawari, K., Mihai, I., Hsiung, P.A., Mishra, R. (eds.) Smart Computational Strategies: Theoretical and Practical Aspects, pp. 69-80. Springer, Singapore (2019). https://doi.org/10.1007/978-981-13-6295-8_7

53. Soltani, M., Chaari, A., Hmida, F.B.: A novel fuzzy C-regression model algorithm using a new error measure and particle swarm optimization. Int. J. Appl. Math. Comput. Sci. 22(3), 617-628 (2012)

54. Stanley, K.O., Clune, J., Lehman, J., Miikkulainen, R.: Designing neural networks through neuroevolution. Nat. Mach. Intell. 1(1), 24-35 (2019)

55. Sun, H., et al.: A parallel self-organizing overlapping community detection algorithm based on swarm intelligence for large scale complex networks. Future Gener. Comput. Syst. 89, 265-285 (2018)

56. Tan, Y., Shi, Y.: Special section on swarm-based algorithms and applications in computational biology and bioinformatics. IEEE/ACM Trans. Comput. Biol. Bioinf. 15(6), 1863-1864 (2018) 
57. Tan, Y., Zhu, Y.: Fireworks algorithm for optimization. In: Tan, Y., Shi, Y., Tan, K.C. (eds.) ICSI 2010. LNCS, vol. 6145, pp. 355-364. Springer, Heidelberg (2010). https://doi.org/10.1007/978-3-642-13495-1_44

58. Tang, H., et al.: Predicting green consumption behaviors of students using efficient firefly grey wolf-assisted k-nearest neighbor classifiers. IEEE Access (2020)

59. Tarkhaneh, O., Isazadeh, A., Khamnei, H.J.: A new hybrid strategy for data clustering using cuckoo search based on mantegna levy distribution, PSO and k-means. Int. J. Comput. Appl. Technol. 58(2), 137-149 (2018)

60. Tuba, E., Mrkela, L., Tuba, M.: Support vector machine parameter tuning using firefly algorithm. In: 2016 26th International Conference Radioelektronika (RADIOELEKTRONIKA), pp. 413-418. IEEE (2016)

61. Tuba, E., Strumberger, I., Bacanin, N., Zivkovic, D., Tuba, M.: Cooperative clustering algorithm based on brain storm optimization and k-means. In: 2018 28th International Conference Radioelektronika (RADIOELEKTRONIKA), pp. 1-5. IEEE (2018)

62. Vrbančič, G., Fister Jr., I., Podgorelec, V.: Swarm intelligence approaches for parameter setting of deep learning neural network: case study on phishing websites classification. In: Proceedings of the 8th International Conference on Web Intelligence, Mining and Semantics, pp. 1-8 (2018)

63. Wang, B., Sun, Y., Xue, B., Zhang, M.: Evolving deep neural networks by multiobjective particle swarm optimization for image classification. arXiv:1904.09035 (2019)

64. Wang, B., Xue, B., Zhang, M.: Particle swarm optimisation for evolving deep neural networks for image classification by evolving and stacking transferable blocks. arXiv:1907.12659 (2019)

65. Wu, Q., Liu, H., Yan, X.: Multi-label classification algorithm research based on swarm intelligence. Cluster Comput. 19(4), 2075-2085 (2016)

66. Yang, X.-S.: Firefly algorithms for multimodal optimization. In: Watanabe, O., Zeugmann, T. (eds.) SAGA 2009. LNCS, vol. 5792, pp. 169-178. Springer, Heidelberg (2009). https://doi.org/10.1007/978-3-642-04944-6_14

67. Yang, X.S., Deb, S.: Cuckoo search via lévy flights. In: 2009 World Congress on Nature \& Biologically Inspired Computing (NaBIC), pp. 210-214. IEEE (2009)

68. Yang, X.S., Gandomi, A.H.: Bat algorithm: a novel approach for global engineering optimization. Eng. Comput. 29(5), 464-483 (2012)

69. Yazdani, M., Jolai, F.: Lion optimization algorithm (LOA): a nature-inspired metaheuristic algorithm. J. Comput. Des. Eng. 3(1), 24-36 (2016)

70. Zedadra, O., Guerrieri, A., Jouandeau, N., Spezzano, G., Seridi, H., Fortino, G.: Swarm intelligence and IoT-based smart cities: a review. In: Cicirelli, F., Guerrieri, A., Mastroianni, C., Spezzano, G., Vinci, A. (eds.) The Internet of Things for Smart Urban Ecosystems. IT, pp. 177-200. Springer, Cham (2019). https://doi.org/10. 1007/978-3-319-96550-5_8

71. Zhang, S., Lee, C.K., Yu, K., Lau, H.C.: Design and development of a unified framework towards swarm intelligence. Artif. Intell. Rev. 47(2), 253-277 (2017). https://doi.org/10.1007/s10462-016-9481-y

72. Zhang, Y., Song, X.F., Gong, D.W.: A return-cost-based binary firefly algorithm for feature selection. Inf. Sci. 418, 561-574 (2017)

73. Zhao, R.Q., Tang, W.S.: Monkey algorithm for global numerical optimization. J. Uncertain Syst. 2(3), 165-176 (2008)

74. Zhao, X., Wang, C., Su, J., Wang, J.: Research and application based on the swarm intelligence algorithm and artificial intelligence for wind farm decision system. Renew. Energy 134, 681-697 (2019) 